\title{
3D visualization of optical ray aberration and its broadcasting to smartphones by ray aberration generator
}

Brandon Hellman, Erica Bosset, Luke Ender, Naveed Jafari, Phillip McCann, et al.

Brandon Hellman, Erica Bosset, Luke Ender, Naveed Jafari, Phillip McCann, Chris Nguyen, Chris Summitt, Sunglin Wang, Yuzuru Takashima, "3D visualization of optical ray aberration and its broadcasting to smartphones by ray aberration generator," Proc. SPIE 10590, International Optical Design Conference 2017, 105900D (27 November 2017); doi: 10.1117/12.2288143

SPIE Event: International Optical Design Conference - IODC 2017, 2017, Denver, United States 


\author{
3D visualization of optical ray aberration and \\ its broadcasting to smartphones by Ray Aberration Generator \\ Brandon Hellman ${ }^{1}$, (alphabetically) Erica Bosset ${ }^{1}$, Luke Ender ${ }^{1,2}$, Naveed Jafari ${ }^{1,3}$, \\ Phillip McCann ${ }^{1}$, Chris Nguyen ${ }^{1}$, Chris Summitt ${ }^{1}$, Sunglin Wang ${ }^{1}$, Yuzuru Takashima ${ }^{1}$ \\ ${ }^{1}$ University of Arizona, 1630 E University Blvd, Tucson AZ, hellman@email.arizona.edu; \\ ${ }^{2}$ Whitworth University, Spokane WA; \\ ${ }^{3}$ University of New Mexico, Albuquerque NM
}

\begin{abstract}
The ray formalism is critical to understanding light propagation, yet current pedagogy relies on inadequate 2D representations. We present a system in which real light rays are visualized through an optical system by using a collimated laser bundle of light and a fog chamber. Implementation for remote and immersive access is enabled by leveraging a commercially available 3D viewer and gesture-based remote controlling of the tool via bi-directional communication over the Internet.
\end{abstract}

Keywords: aberrations, ray, raytrace, visualization, teaching tool, geometrical optics

\title{
1. INTRODUCTION
}

The ray is a conceptual representation of the path along which electromagnetic energy propagates. The concept creates an essential, common language in the imaging and optical system design community. Rays propagated through optical systems offer an extremely rich basis of information, covering items such as geometrical attributes, image and object locations, and system performance. ${ }^{1}$ Ray conveys even richer information which is not limited to first order quantities, but on aberrations via ray trajectory as well as power arrangement of system. For example, tracing rays through a 30-element lithographic lens will show an extremely smooth flow of rays. ${ }^{2}$ Optical design experts recognize how a smooth flow of rays, with bulge and waist constructions, is crucial to minimizing aberrations. In practice, observing ray trajectories and relating the information to system performance is the foundation of optical system design.

Although lens design experts understand the importance of the information derived from ray paths, effective methods to demonstrate and visualize ray propagation through optical systems have not been fully investigated, especially for educational purposes. Textbooks and simulated renderings on screen are available teaching resources, though real-world demos are lacking.

Figure 1(a) and (b) depicts on-axis spherical aberration for a singlet. Students often encounter such diagrams while studying literatures. These types of diagrams are used to assist students in visualization the physical manifestation of optical aberrations. ${ }^{3}$

International Optical Design Conference 2017, edited by Peter P. Clark, Julius A. Muschaweck,

Richard N. Pfisterer, John R. Rogers, Proc. of SPIE-OSA Vol. 10590, 105900D - C 2017 SPIE

CCC code: $0277-786 \mathrm{X} / 17 / \$ 18 \cdot$ doi: $10.1117 / 12.2288143$ 
a)

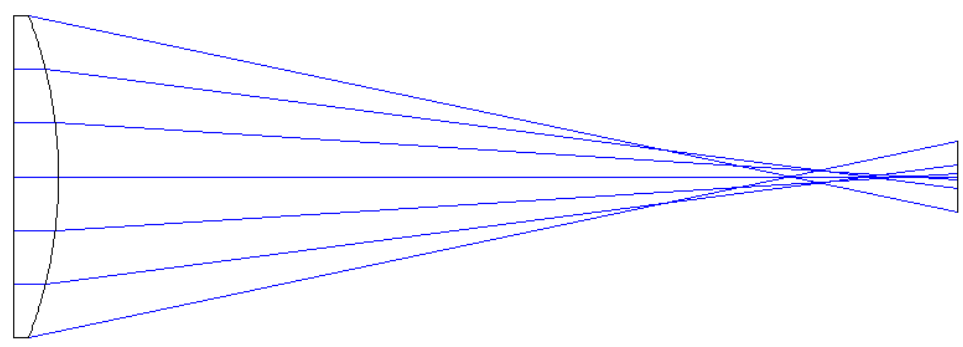

b)

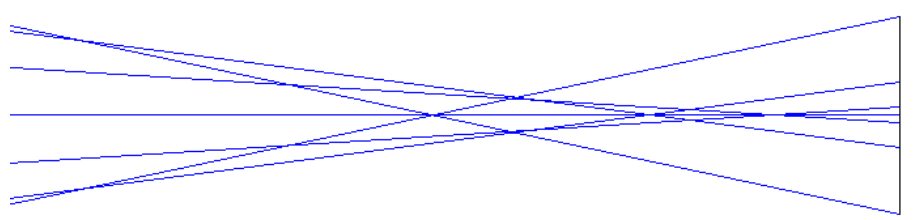

Figure 1: (a) Positive singlet with spherical aberration. (b) Close-up of the caustic.

Though these diagrams may be clear for a seasoned scientists and engineers in the optics field, the significance can easily be lost on students new to optics. For instance, the rays in the diagram clearly converge, but most people do not attribute light to rays in the real world. The most hands-on experience many budding students get is often focusing sunlight with a magnifying glass, but the only visible aspects are the sun, the lens, and the focused spot. The entire converging bundle is not apparent to the observer, let alone individual rays. This leaves a significant disconnect between introductory visualizations and real-world physics.

To fill the gap of missing physical demonstrations of optical aberrations, Roland Shack used a series of strings to create a physical manifestation of astigmatism, clearly demonstrating through-focus effects. Though this is a 3D, real-world object, and though taught strings are decent representations of light rays, the system does not use actual light and it is not dynamic.

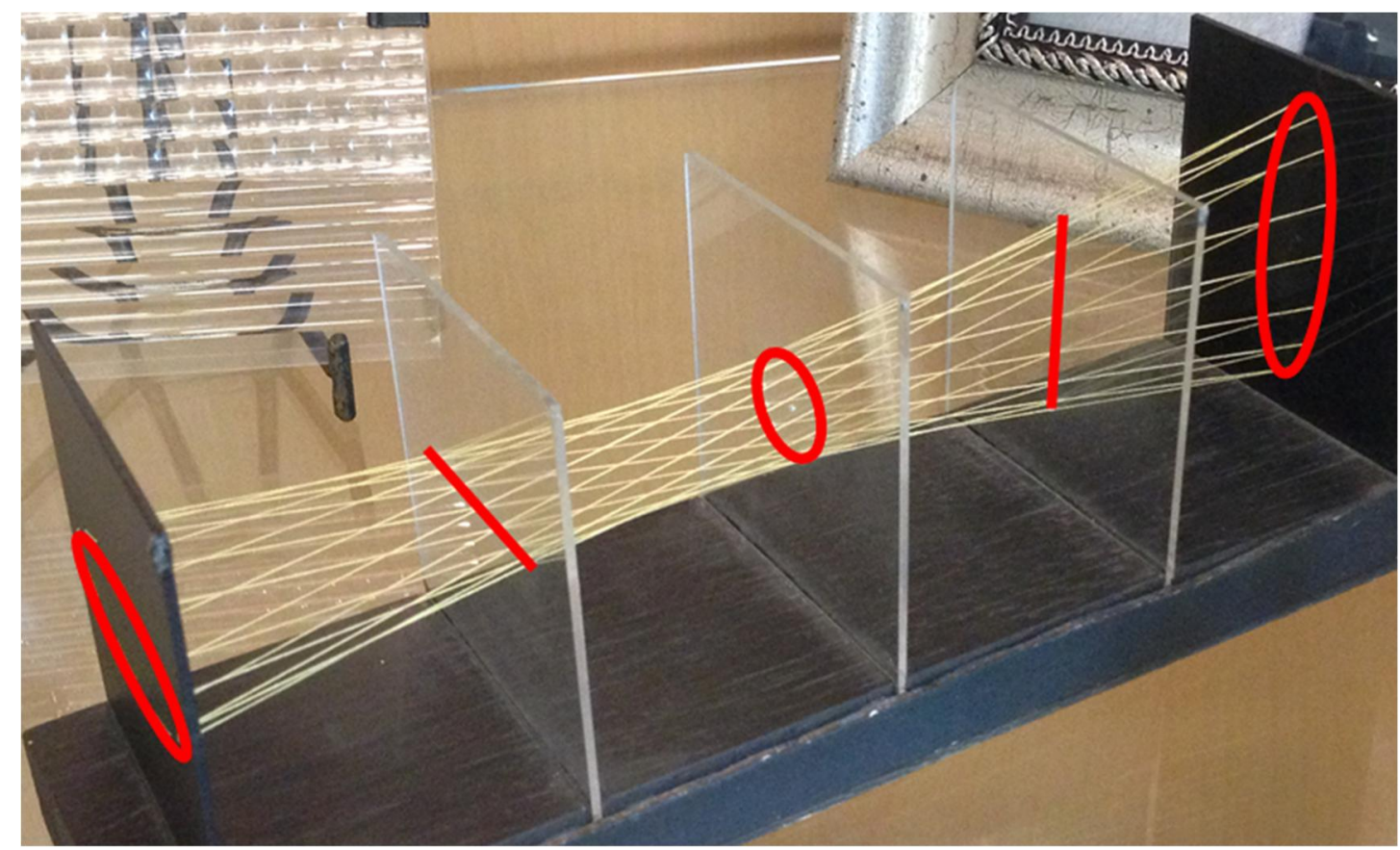

Figure 2: Roland Shack's real-world model of astigmatism, with superimposed ray-bundle outlines. ${ }^{4}$ 
Chris Summitt, at University of Arizona, previously used a special, long-exposure technique to capture complete light paths. The photograph of a diffraction pattern from a pinhole assembly was taken by moving a screen quickly across the optical path of the beam during an exposure for a short period of time compared to the full exposure time. This technique is similar to the way a $3 \mathrm{D}$ visualization of an object was created by a projector and a fast rotating screen. ${ }^{5}$ Though these photos are not live or a real-world effect, the captures are an impressive technique to demonstrate and fully describe light propagation through an optical system.

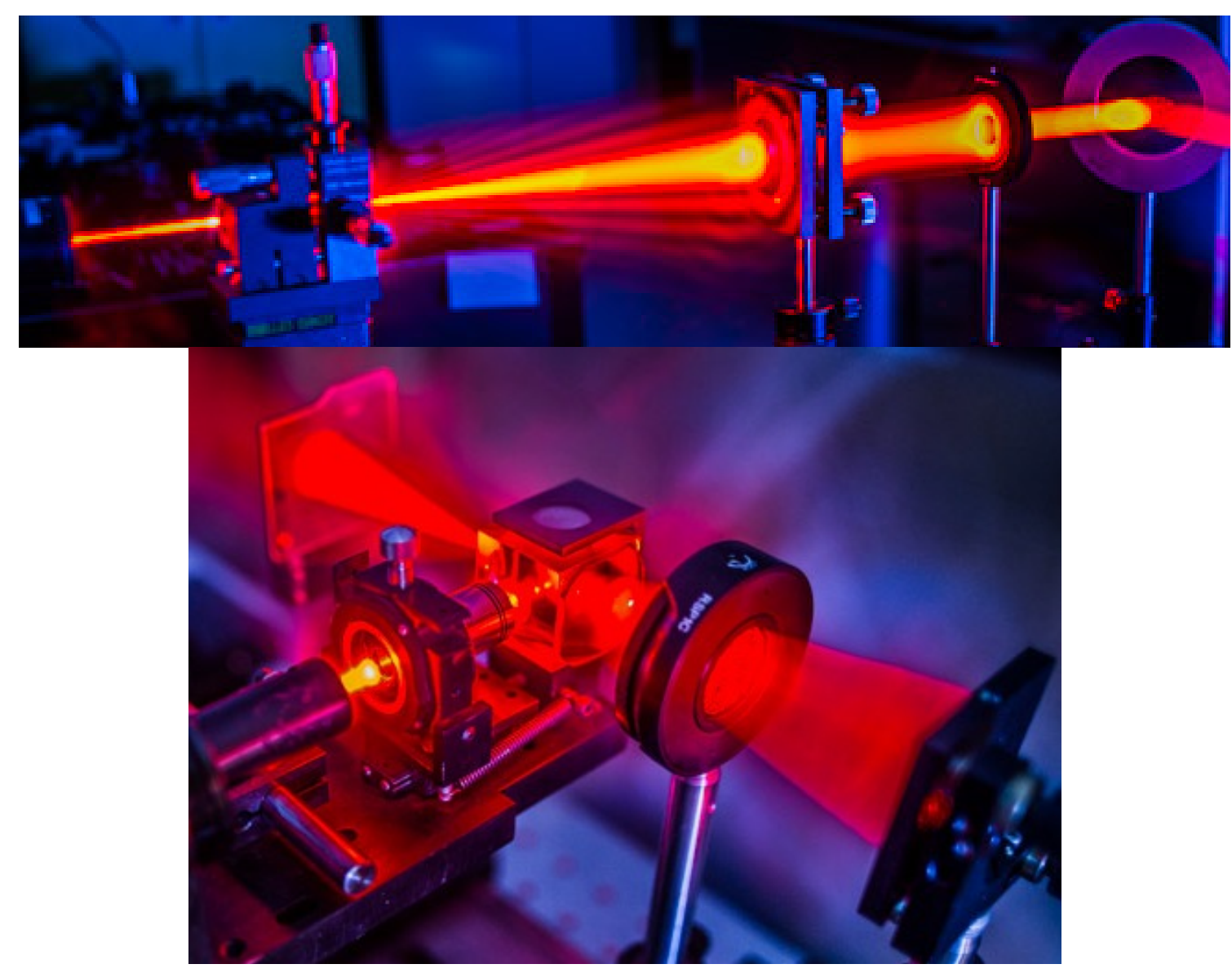

Figure 3: Chris Summitt's captures of spatial filtering of a beam (top), and focusing through a Shack cube in a Fizeau interferometer (bottom).

Early $20^{\text {th }}$ century particle physics also needed new tricks to display physical phenomena which cannot typically be visually observed. In 1911, C. T. R. Wilson presented a method to visualize alpha and beta particles by condensing water on the particles within a water-vapor-filled "cloud-chamber". ${ }^{6}$

By a similar "cloud-chamber" technique, we present a system which allows the visualization of light rays propagating through an optical system. The Ray Aberration Generator (RAG) is a teaching tool to demonstrate ray propagation and real-world manifestations of third-order aberrations, including spherical aberration, coma, astigmatism, field curvature, and distortion. ${ }^{7}$

\section{CREATION, CONTROL, AND VISUALIZATION OF RAYS}

Tangential and sagittal ray fans are created by two $\sim 1 \mathrm{~mm}$ collimated laser beams. A green laser is sent through a 1D diffraction grating to create a set of narrow rays along the diverging from one another in the $\mathrm{x}$-dimension, but maintaining each beams' collimation. The setup is repeated for a red laser splitting across the y-dimension. The optical axes are combined by a beam splitter, and the numerous diverging beams are made co-parallel by a single lens module. 


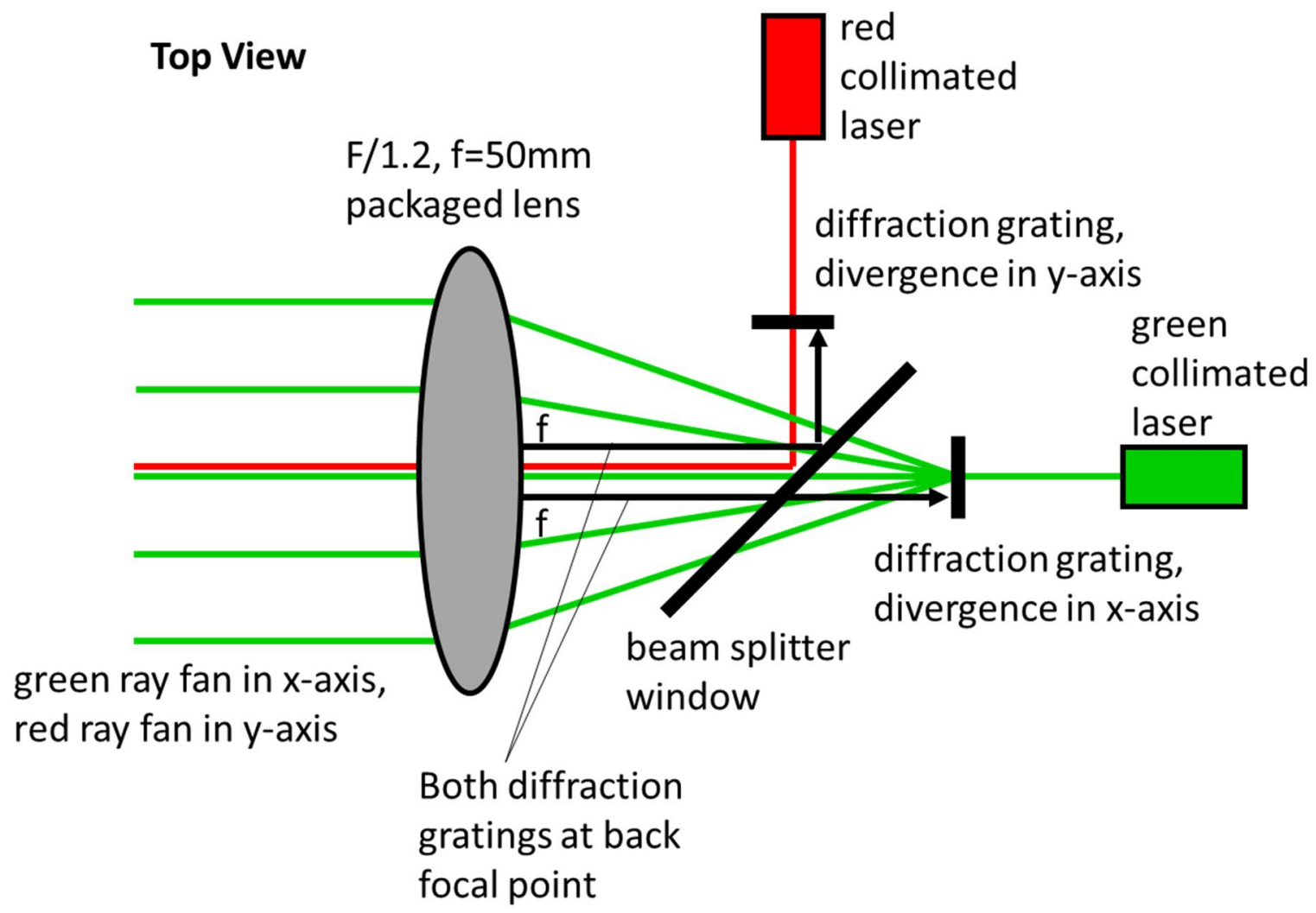

Figure 4: Schematic of ray fan creation, top view. Green, x-axis ray fan shown. Red, y-axis ray fan in orthogonal dimension not shown.

The several $\sim 1 \mathrm{~mm}$, collimated beams act as individual rays being traced through a system. Each beam maintains its collimation well enough for the demo due to its narrow diameter with respect to the whole aperture of the optical system under test. The test system does greatly impact the rays' angles and positions with respect to each other, creating the ray trace effect.

The ray incident angles are controlled by a folding mirror located in front of the fog-filled tank containing a lens. The angle and height of the folding mirror is digitally controlled by two motors $\left(y\right.$ and $\theta_{y}$ ) to select the input rays' field angle $H$ (primarily dependent on mirror angle), and pupil coordinate $\rho$ (primarily dependent on mirror height). 
a)

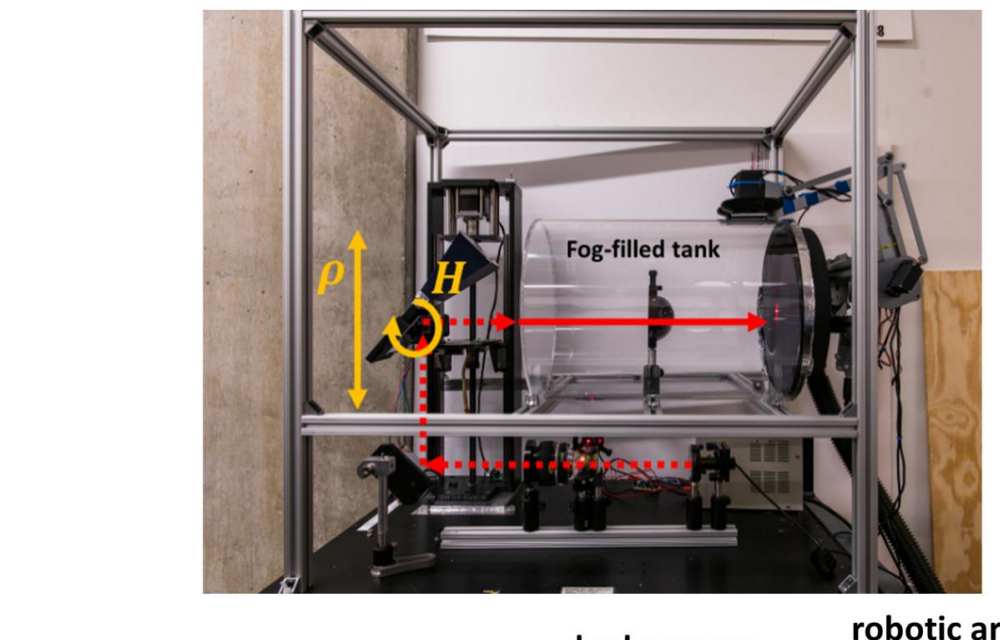

b)

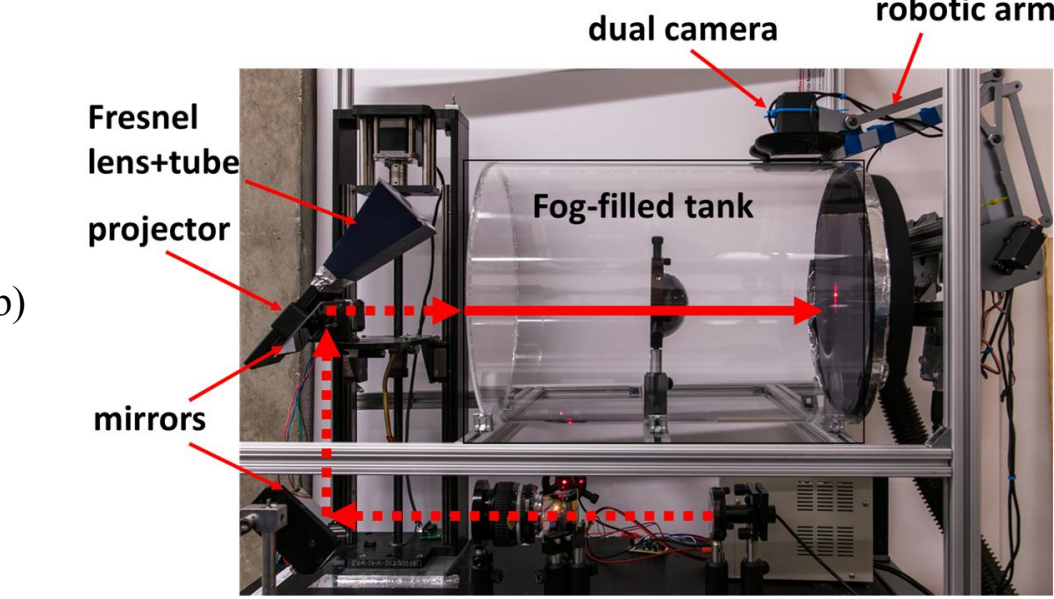

Figure 5: a) Motorized ray selection. Solid red ray denotes when the ray path becomes visible to the observer by scatter. b) Labeled components.

A secondary illumination system is implemented by collimating the exit pupil of a handheld laser raster-scan projector (Sony MP-CL1A) with a Fresnel lens. The collimated projector is attached to the back of the motorized coupling mirror. If the projector is used instead of the lasers, the motor controls can be used to select $H$ and $\rho$, and the rays fan structure can be modified digitally by the projector output.

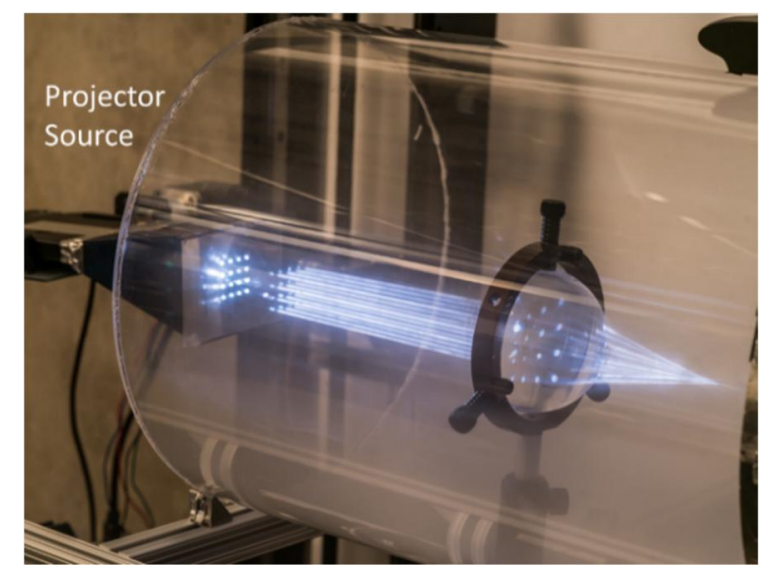

Figure 6: Handheld laser projector, with collimated exit pupil by Fresnel lens, as the source. 
As each ray propagates through the tank, light is scattered by the fog which allows visualization of each ray path. We used an acrylic hemispherical lens $75 \mathrm{~mm}$ in diameter. The material of the lens is imperfect, causing scatter as the ray propagates through the lens, allowing visualization of the ray path within the lens itself. The entire path of each ray can be visualized from the entrance surface to the final image plane. The left image in Figure 7 shows the sources (bottom) coupling into the tank (top) by the mirrors (left).
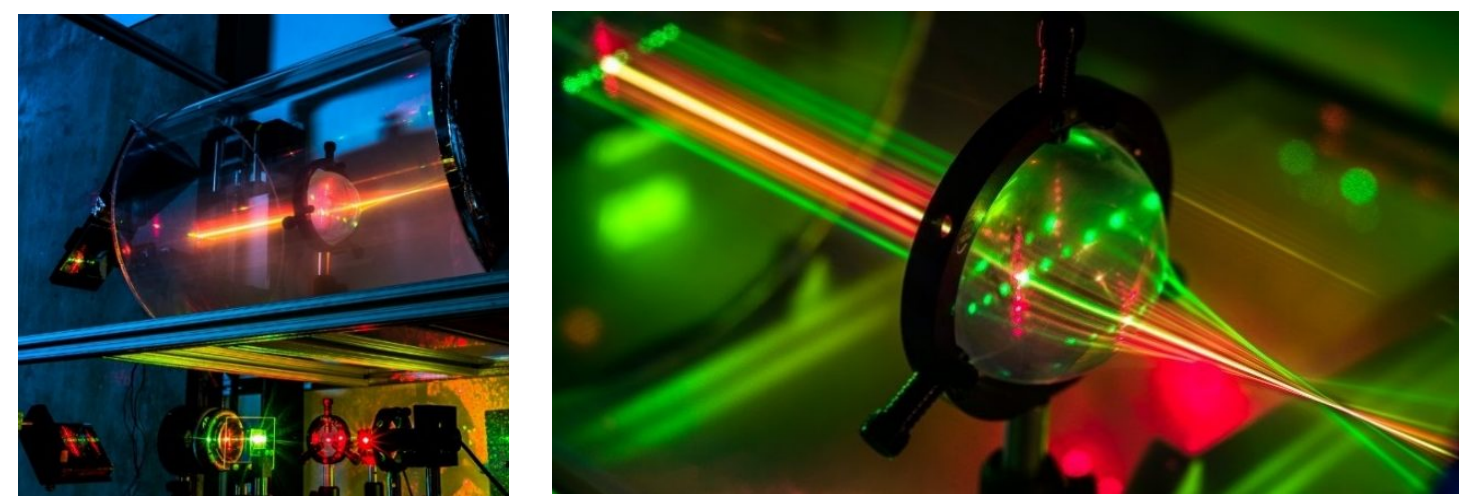

Figure 7: RAG platform (left) and ray tracing (right).

The lens, which is interchangeable, has defined aberrations due to the surface shapes. To view different ray effects of the third order aberrations, the input ray trajectories can be adjusted through software controlling a motorized input coupling mechanism. The system has control of both field height and pupil coordinate.

\section{REAL-WORLD RAY TRACE}

The array of collimated beams is a very effective ray trace due to the small beam diameter and pitch. Though some spreading of an individual beam may occur due, Figure 8 shows how the injected beams match the simulated rays.
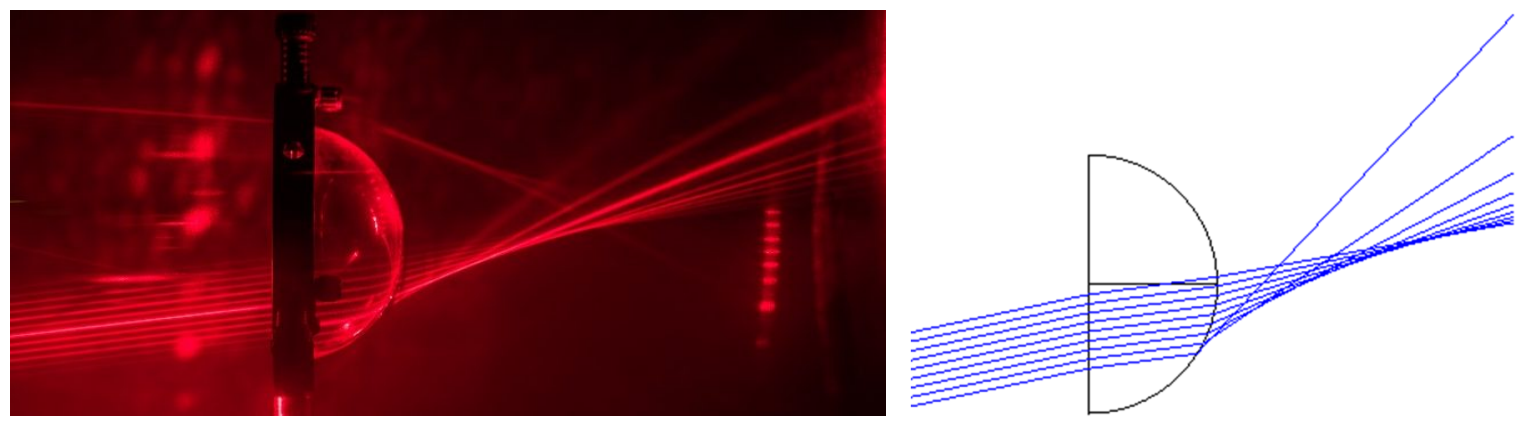

Figure 8: RAG (left) comparison to Zemax model (right).

The transverse ray error for spherical aberration is

$$
\varepsilon_{y}=-2(F / \#) W_{040} y_{p}^{3}
$$

where $W_{040}$ is the amount of spherical aberration in the system design, $y_{p}$ is the y-axis pupil coordinate, and $F / \#$ is the system F-number. ${ }^{8}$ It is clear in both the RAG demo ray trace and the simulated ray trace that the rays injected closer to the edge of the lens have significantly greater transverse error at the focal plane. The cubic functionality of the transverse ray error for spherical aberration is also apparent since the rays are parallel and linearly-spaced at the first surface of the lens. 
The RAG can be used to view effects of all third-order aberrations: spherical aberration, coma, astigmatism, field curvature, and distortion. An image plane is also observable, of particular interest for distortion. The paths distinctly map ray distributions across planes, showing the creation of the distortion effect as seen in Figure 9.

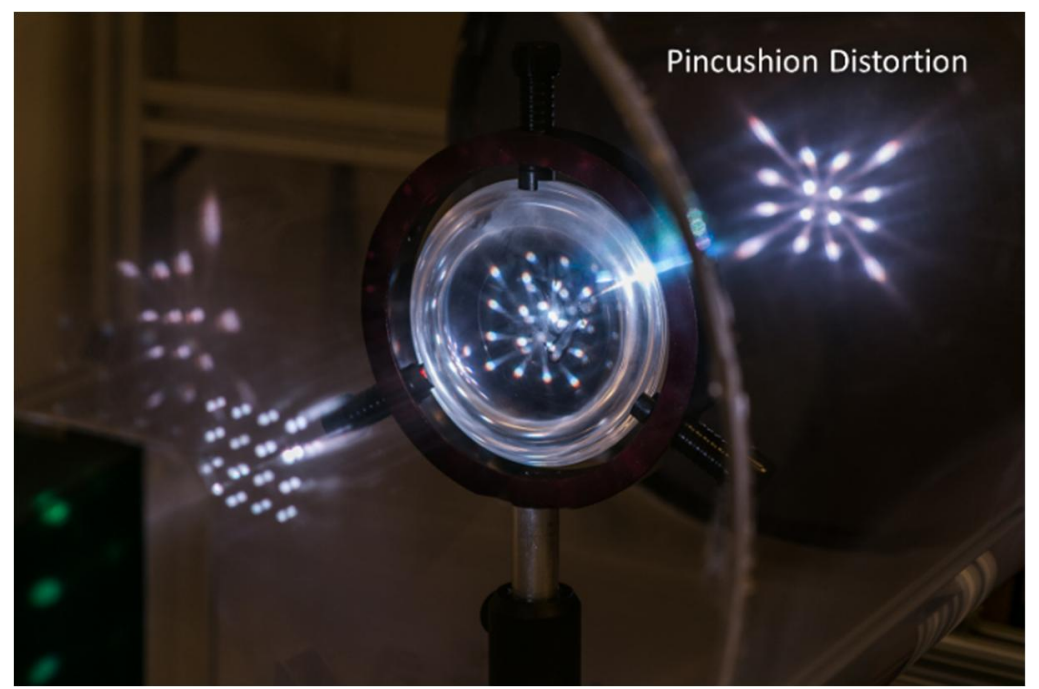

Figure 9: Distortion mapping across planes, from source (left), to lens (center) to image plane (right). Grid ray bundle created by projector.

\section{REMOTE ACCESS}

The system is not portable, but a method was developed to grant students around the world access to the RAG. A robotic arm with a stereoscopic camera captures a 3D video to stream to a smartphone. Using Google Cardboard, a do-it-yourself cardboard headset which turns a smartphone into a virtual reality display, remote users can view the 3D demo live. Head gesturing in an Android app sends accelerometer data back to the robotic arm, moving the camera accordingly. The head gesturing, which causes live perspective shifting, creates a more immersive 3D experience.

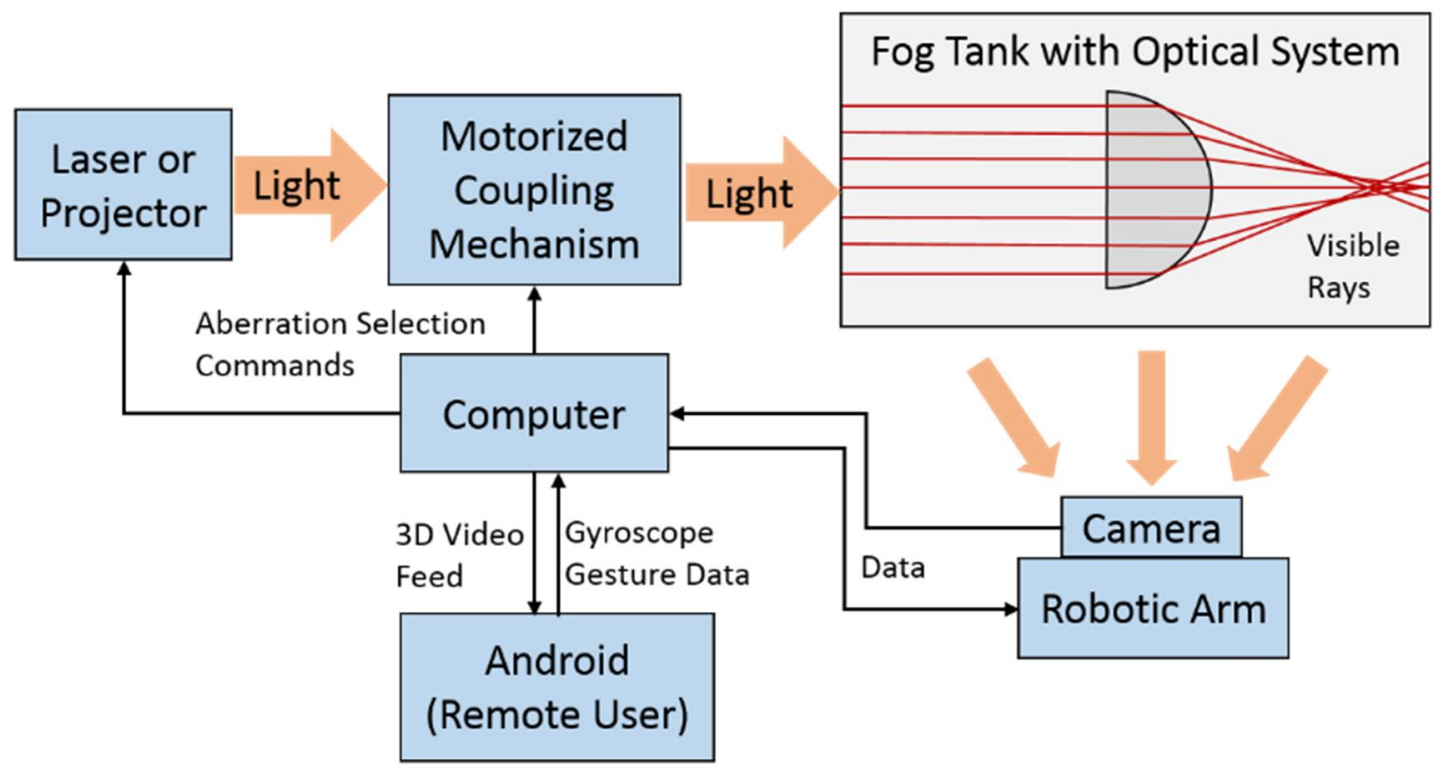

Figure 10: RAG Block Diagram 


\section{FUTURE WORK}

An expansion of this work is ongoing, including interfacing between the RAG and Code V, a well-known optical design software. The goal of the next-generation Ray Aberration Generator is to demonstrate a physical ray trace in parallel to a corresponding ray trace simulation with equivalent adjustable parameters in software. A single input from a local or remote user will initiate both systems to adjust to prescribed input rays. The parallel ray traces will offer a direct bridge between computer simulation and physical manifestation.

Additionally, the RAG will allow for dynamic system building by using a set of interchangeable, 1" catalogue lenses on a modular lens mounting system with adjustable spacings. This modular system will allow students degrees of freedom within the system design, allowing system optimizations to be visualized alongside the optical design software.

\section{CONCLUSIONS}

The Ray Aberration Generator offers a new real-world platform to demonstrate ray propagation and aberrations using real light rays. The interchangeable optical system, programmable input rays, and immersive remote access offer extensive capabilities to help students visualize and understand light ray propagation.

\section{REFERENCES}

[1] Sasián, J., [Introduction to Aberrations in Optical Imaging Systems], Cambridge University Press, Cambridge, 13 (2012).

[2] Bruning, J. H., “Optical lithography: 40 years and holding,” Proc. SPIE 6520, 652004 (2007).

[3] Kingslake, R. and Johnson, R. B., [Lens Design Fundamentals, second edition], Academic Press, Cambridge, 116 (2010).

[4] Roland Shack's String Model of Astigmatism. Located in the Museum of Optics, College of Optical Sciences, University of Arizona. Photo by Brandon Hellman on Sept 20, 2016.

[5] Jones, A., McDowall, I., et al, "Rendering for an Interactive 360 Light Field Display," ACM, SIGGRAPH (2007).

[6] Wilson, C. T. R., "On a Method of making Visible the Paths of Ionising Particles through a Gas," Proc. Roy. Soc. of London, Series A, Vol. 85, No. 587, 285-288 (1911).

[7] Summitt, C., "Spherical Aberrations," OPN, photo in "After Images", 16-02-01, 28 Jan 2016.

[8] Greivenkamp, J.E., [Field Guide to Geometrical Optics], SPIE, Bellingham, pp 71, 75 (2004). 